\title{
Future Perspectives, Education and Training of Flexible Ureteroscopy for Urolithiasis-Some Personal Views
}

\author{
Musab Umair Khalid* \\ Armed Forces Institute of Urology, Pakistan
}

*Corresponding author: Musab Umair Khalid, Armed Forces Institute of Urology,

Rawalpindi, Pakistan, Tel: +923456969731; Email: musabumair923@gmail.com

\section{Editorial \\ Volume 4 Issue 2}

Received Date: April 05, 2019

Published Date: April 09, 2019

DOI: $10.23880 /$ oajun-16000161

\section{Abstract}

Urolithiasis is a common problem affecting patients irrespective of age and gender. Importantly, it is a recurrent disease with a lifetime risk of recurrencureteroscopygh. In the present days, almost all urinary tract stones were treated in a completely noninvasive fashion. This editorial describes the technical progression, modern use, and future perspective of ureteroscopy (URS) for stone diseases. Advantages of URS are minimal contraindications, easy access to the upper tract, fewer complications and shorter length of stay in the hospital. With ever decreasing scope size and better optics coming to market no corner of the urinary tract is inaccessible with URS. A large area of attention needs to be placed not only on stone prevention, with simple but effective patient education and lifestyle interventions but also on the education and training of a future generation of doctors.

\section{Introduction}

Urolithiasis is a term used to describe calculi or stones that form in the urinary tract. The risk of urolithiasis increases as a result of any factor that leads to urinary stasis due to a reduction or obstruction of urinary flow. In Asia, about $1 \%-19.1 \%$ of the population suffers from urolithiasis [1]. The worldwide prevalence, incidence, and composition of calculi vary and have changed in the last several decades, with prevalence ranging from $7 \%$ to $13 \%$ in North America, 5\%-9\% in Europe, and 1\%-5\% in Asia [2].

Ureteroscope is a small flexible telescope which is inserted into the bladder and ureter. Ureteroscopy is a minimally invasive technique which not only minimizes postoperative complications but also provides easy and efficacious access to the upper urinary tract. It has become the first-line option for treatment of ureteral and renal stones worldwide with high success rates and low morbidity profile.

It is now considered as the standard procedure in all endourology clinics. Although the entire urologists is now familiar with and, probably perform ureteroscopy, it is always possible to do it in a better way. This development is not only due to technological advancements but also to the routine use of the Holmium: YAG LASER for intracorporeal lithotripsy, capable of destroying any stone regardless of its composition or location, surpassing the ability of any other lithotripter [3]. There have been many new developments including new endoscopy design, more effective auxiliary tools, improvement in management protocols, and the introduction of robotic technology, combining both ureteroscopy and percutaneous nephrolithotomy, improvement in laser technology, and so on. Ureteroscopy usually is an outpatient procedure however; patients may require an 


\section{Open Access Journal of Urology \& Nephrology}

overnight hospital stay if the procedure proves lengthy or difficult. There are two ways to perform ureteroscopy for stones. If the stone is small, Urologist will insert a scope into the ureter to remove the stone. The scope used in this procedure will have a basket at the end of a wire that is run through an extra channel in the ureteroscope to collect the stone. If the stone is larger, Urologist will extend a flexible fiber through the scope up to the stone. With a laser beam shining (Lithoclast) through the scope, it will break the stone into pieces small enough to be passed out of the body with urine.

Ureteroscopy is important to diagnose and manage complex urolithiasis cases. The aim of this editorial is to highlight an important area of interest in endourology which will open new gates of education and research in developing countries. From consultant to a post-graduate resident, involvement in endourology is not only desirable but is increasingly becoming compulsory. Many of the open urological operations are now obsolete in developed countries due to the advent of ureteroscopy. It needs special consideration in developing countries due to its efficacy and lesser complications.

\section{Ending Note}

Ureteroscopy is a fundamental part of modern Urological practice. The aim of minimally invasive urology is to improve management strategy along with minimal surgical complications. Ureteroscopy is part of clinical research, diagnosis and management and at the heart of all Urological advances. It looks at new ways to prevent, detect, or treat disease. It looks at other aspects of care, such as improving the quality of life for people with chronic urological illnesses.

\section{References}

1. Liu Y, Chen Y, Liao B, Luo D, Wang K, et al. (2018) Epidemiology of urolithiasis in Asia. Asian journal of urology 5(4): 205-214.

2. Sorokin I, Mamoulakis C, Miyazawa K, Rodgers A, Talati J, et al. (2017) Epidemiology of stone disease across the world. World journal of urology 35(9): 1301-1320.

3. Santos JM (2018) Ureteroscopy from the recent past to the near future. Urolithiasis 46(1): 31-37. 